\title{
Die Methode der Linienmomente zur konturbasierten Echtzeit- Bildverarbeitung und industrielle Anwendungen
}

\author{
Georg Lambert, Darmstadt
}

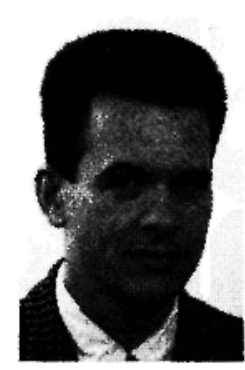

\begin{abstract}
Dipl.-Ing. Georg Lambert ist wissenschaftlicher Mitarbeiter am Institut für Regelungstechnik der Technischen Hochschule Darmstadt, FG Regelsystemtheorie \& Robotik (Leiter: Prof. Dr. rer. nat. Dipl.-Ing. Henning Tolle). Er beschäftigt sich mit der Untersuchung und Entwicklung von Algorithmen zur Oberflächeninspektion in Echtzeit, konturbasierter Bildverarbeitung, Verfahren der Texturanalyse sowie Architekturen von EchtzeitSoftwaresystemen zur Bildverarbeitung.

Adresse: TH Darmstadt, Institut für Regelungstechnik, FG Regelsystemtheorie \& Robotik, Landgraf-GeorgStraße 4, D-64283 Darmstadt, Tel.: (06151)16-3542, Fax: (06151)16-2507, E-Mail: lamb@rt.e-technik.thdarmstadt.de
\end{abstract}

Die industrielle Qualitätssicherung mit Bildverarbeitungssystemen stellt hohe, zeitkritische Anforderungen an automatische Inspektionssysteme. Der vorliegende Artikel stellt einen neuartigen Ansatz, basierend auf einer Echtzeit-Konturextraktion und Polygonapproximation mit anschließender schneller Algorithmik zur Verarbeitung der Konturdaten vor. Ausgehend von den bekannten Flächenmomenten wird die Formulierung für Konturen in Form von Linienmomenten vorgenommen. Der Linienmomentenansatz bietet dabei die Möglichkeit, nicht zusammenhängende Konturfragmente ohne geschlossenen Rand als Einheit zu modellieren und bietet damit weitaus mehr Flexibilität als andere konturbasierte Methoden. Auf die schnelle Berechnung der Linienmomente wird eingegangen. Erweiterungen des Linienmomentenansatzes auf verschiedene Momententypen ermöglichen die Berechnung einer Vielzahl von Invarianten bezüglich Translation, Skalierung und Rotation. An zwei Anwendungen wird die Güte der verschiedeñen Linienmomenten-Invarianten untersucht und die Erkennungsleistung der Methode herausgestellt.

The method of line moments for contour based real time image analysis and industrial applications

Strong and time critical requirements have to be met by machine vision systems for industrial quality control tasks. This article proposes a new approach, based on real time contour extraction and polygon approximation followed by fast algorithms for the evaluation of the contour data. Based on the known area moments line moments are derived for contour data. The line moment approach inherently provides the possibility to model unconnected contour fragments and therefore incorporates much more flexibility than other contour based methods. The fast determination of line moments is presented. The expansion of the line moment approach to other moment types enables the calculation of a variety of invariants with respect to translation, scale and rotation. On two applications the significance of the different line moment invariants and the recognition performance of the method are emphasized.

\section{Einleitung}

Für die Automatisierung von Qualitätssicherungsaufgaben werden in zunehmendem Maße Bildverarbeitungssysteme erfolgreich eingesetzt. Die vollständige Qualitätskontrolle an der laufenden Produktion wird wegen der Produkthaftungsgesetzgebung und den Qualitätsanforderungen an moderne Produkte angestrebt. Durch die ständig steigenden Produktionsgeschwindigkeiten werden an automatische Inspektionssysteme äußerst zeitkritische Anforderungen gestellt. Die Geschwindigkeitssteigerungen in der Mikroelektronik stehen dabei in ständiger Konkurrenz zu dem Fortschritt bei den Produktionsmaschinen.

Durch die teilweise extremen Zeitanforderungen an automatische Inspektionssysteme wird vielfach unflexible Spezialhardware zur Lösung einzelner Aufgabenstellungen eingesetzt. Im vorliegenden Beitrag wird ein

Bild 1: Architektur zur flexiblen, konturbasierten Echtzeit-Bildverarbeitung, basierend auf dem Linienmomentenansatz.

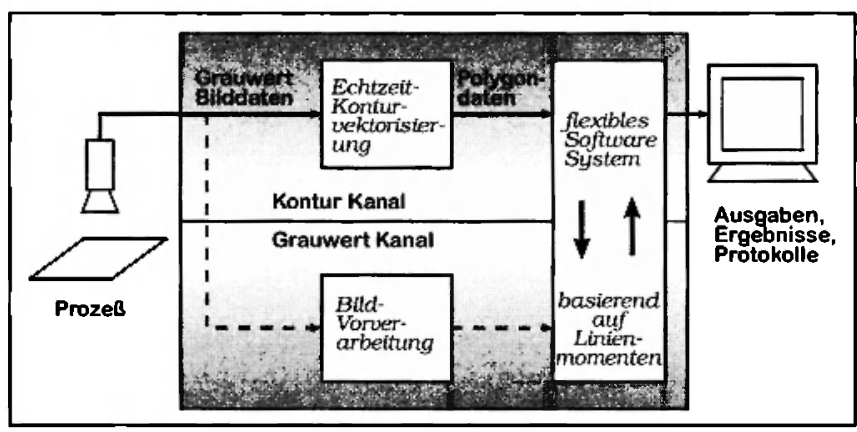




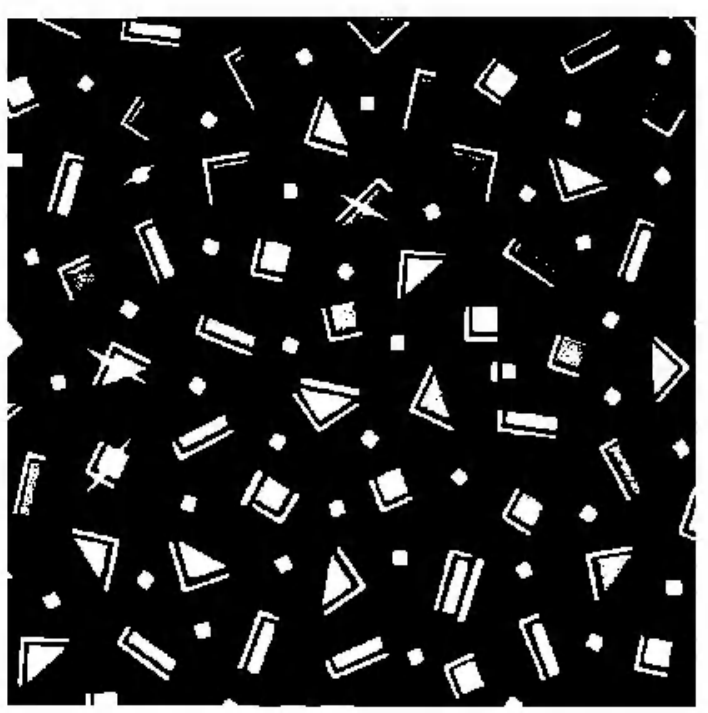

Bild 2: Grauwertszene einer fehlerhaften, mit Mustern bedruckten Folie. $(512 \times 512$ Bildpunkte, Bildausschnitt ca. $30 \mathrm{~cm} \times 30 \mathrm{~cm}, 8$ fehlerhafte Texel.)

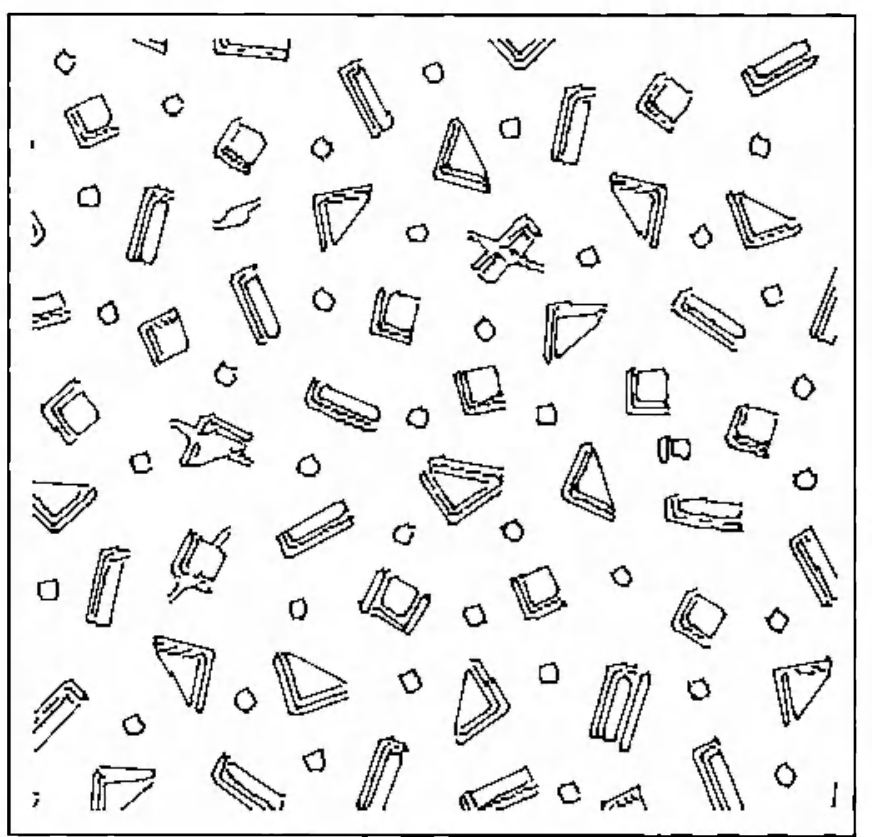

Bild 3: Aus den Polygondaten rekonstruiertes Konturbild der Szene aus Bild 2. (2789 Polygoneckpunkte in 538 Konturfragmenten.)

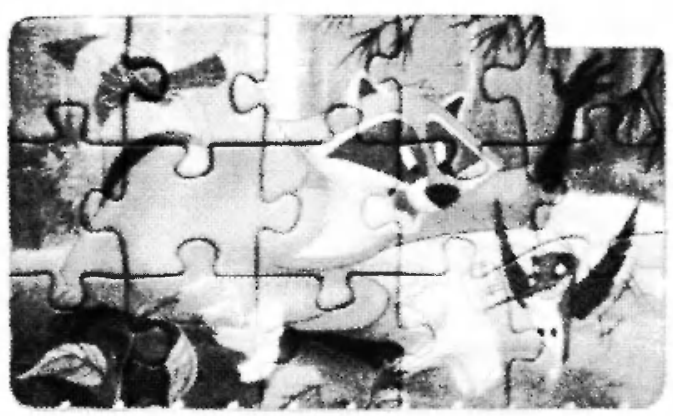

Bild 4: Das zusammengesetzte Puzzle, bestehend aus 15 Teilen.

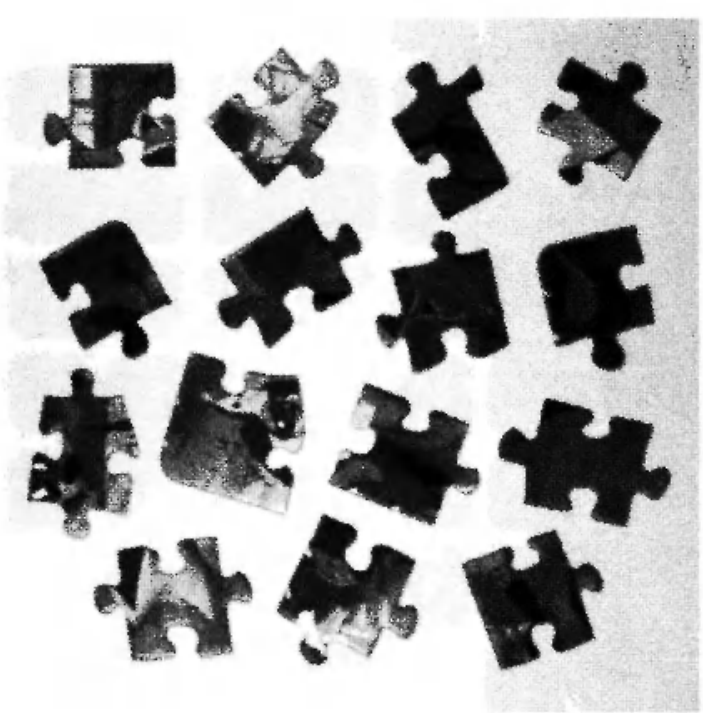

Bild 5: 15 Puzzleteile in beliebigen Lagen als Beispiel zur 2DObjekterkennung.

neuer konturbasierter Ansatz zur Echtzeit-Bildverarbeitung vorgestellt. Die zugrundeliegende Systemarchitektur, wie in Bild 1 dargestellt, bietet einen Kompromiß zwischen schneller Hardware und flexibler Software. Die Konturextraktion und Approximation durch Polygone wird als in Echtzeit realisierbare Standardaufgabe der Bildverarbeitung angesehen, deren Implementierung in Spezialhardware (wie für die in diesem Aufsatz aufgeführten Beispiele) oder auf Signalprozessoren erfolgen kann. Die Methode der Linienmomente (siehe auch $[1 ; 2]$ ), die Inhalt dieses Beitrags ist, stellt ein flexibles, neuartiges Verfahren dar, das als Softwarelösung die Polygondaten des Echtzeit-Konturvektorisierers effizient verarbeiten kann.

Die Konturextraktion ist immer dann sinnvoll einsetzbar, wenn die zu analysierende Szene strukturiert ist, also Grauwertkanten eindeutig extrahierbar sind. In diesem Fall führt die Konturextraktion und Polygonapproximation zu einer drastischen Reduktion des Datenstromes bei nahezu vollständigem Informationserhalt. Im vorliegenden Beitrag werden Anwendungen zur Inspektion von Texturen mit Texturelementen (Texeln) sowie zur schnellen 2D-Objekterkennung betrachtet. Die zu durchlaufenden Verfahrensschritte sowie die Theorie werden daher an diesen Beispielen, die sich durch den gesamten Artikel ziehen, motiviert. Bild 2 zeigt die Grauwertszene einer zu inspizierenden, mit Mustern bedruckten, fehlerhaften Folie, die als Textur aus Texeln aufgefaßt werden kann. Für den Bereich der 2D-Objekterkennung wurde als Beispiel das in den Bildern 4 und 5 dargestellte Puzzle ausgewählt, da hier die Vorzüge des Linienmomentenansatzes deutlich aufgezeigt werden können.

\section{Konturobjekte}

Die Bilder 3 und 6 zeigen die Konturbilder der in den Bildern 2 und 5 dargestellten Grauwertszenen. Die Konturbilder stellen eine Rekonstruktion aus den 


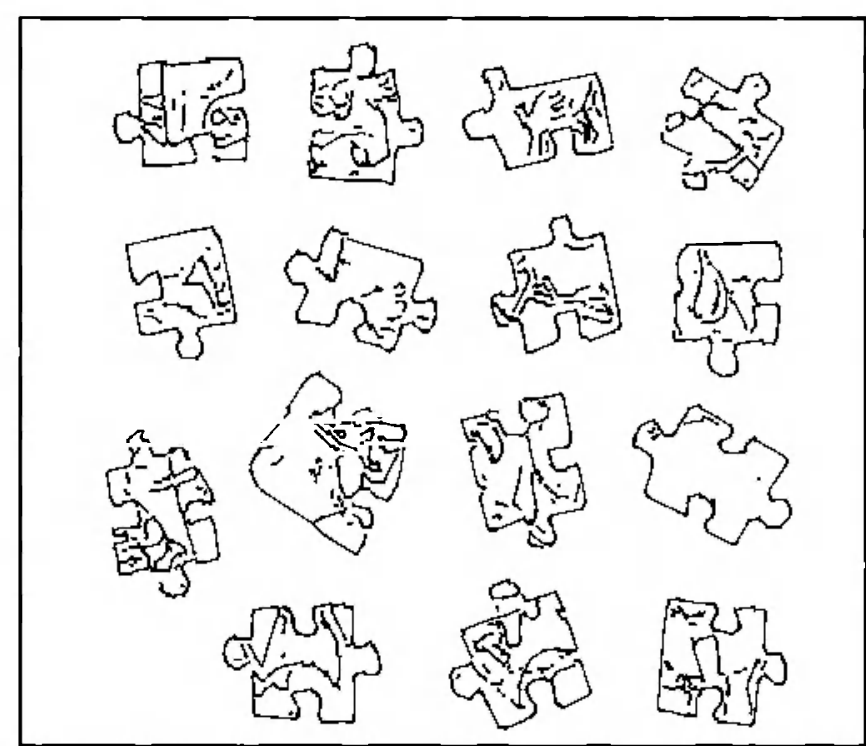

Bild 6: Aus den Polygondaten rekonstruiertes Konturbild der Szene aus Bild 5. (2996 Polygoneckpunkte in 592 Konturfragmenten.)

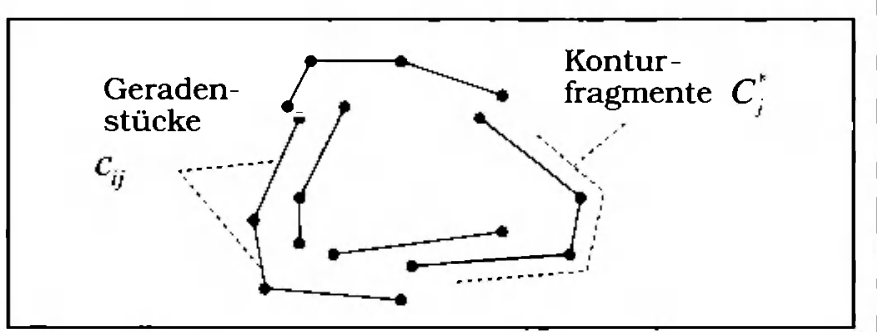

Bild 7: Zur Definition von Konturobjekten.

Polygondaten dar, die von der Echtzeit-Konturvektorisierung (vergleiche Bild 1) in Form von indizierten (mit einem Konturindex), sortierten Punktlisten geliefert werden ${ }^{1}$.

Wie in den Bildern 3 und 6 zu erkennen ist, zerfallen die Konturen einzelner Objekte in zahlreiche Konturfragmente. Geschlossene Ränder liegen nicht vor. Durch die Oberflächenstruktur der Puzzleteile aus Bild 6 ist das Auffinden geschlossener Konturen auch durch eine Nachverarbeitung nicht möglich. Die Stärke der Konturfragmentierung ist zwar vom Konturextraktionsverfahren abhängig, das prinzipielle Problem, mehrere Konturelemente einem Objekt zuordnen zu wollen, steht jedoch allgemein zur Lösung an.

Die Methode der Linienmomente besitzt inhärent die Möglichkeit, beliebige Sammlungen von Konturfragmenten als eine Einheit zu modellieren. Es kann daher auf rechenaufwendige Vorverarbeitungsschritte verzichtet werden. Folgende, flexible Definition von Konturobjekten (vergleiche Bild 7) wird gegeben. Das Konturobjekt $C$ ergibt sich als Sammlung beliebiger Konturfragmente $C_{j}^{*}$, die sich ihrerseits als Mengen von Geradenstücken $c_{i j}$ ergeben:

$$
C=\bigcup_{j} C_{i}^{*}, \quad C_{j}^{*}=\bigcup_{i} c_{i j}
$$

\footnotetext{
'Für die Konturvektorisierung wurde die Spezialhardware VECTEX der Fa. ELTEC Elektronik GmbH, Mainz, eingesetzt.
}

Das Konturobjekt $C$ kann also dargestellt werden als Sammlung beliebiger Geradenstücke:

$$
C=\bigcup_{j} \bigcup_{i} c_{1 j} \text {. }
$$

\subsection{Beispiel eines Segmentieralgorithmus}

Obige Definition von Konturobjekten läßt dem Benutzer alle Freiheiten, nach welchen Kriterien Konturfragmente zu logischen Objekten zu gruppieren sind. Im folgenden soll ein Beispiel eines regelbasierten Segmentieralgorithmus gegeben sowie dessen besonders effiziente Implementierung angesprochen werden. Die Regel, nach der Konturfragmente gruppiert werden, lautet:

Alle lokal benachbarten Konturfragmente sind Teil des selben Konturobjekts.

Anhand von Bild 8 soll der Algorithmus näher erläutert werden. An den Polygoneckpunkten wird in lokalen Fenstern nach benachbarten Konturfragmenten gesucht. Werden solche entdeckt, wird eine logische Gruppierung zu ein und demselben Objekt vorgenommen. Nicht gruppierte Konturfragmente eröffnen ein neues Objekt. Teilgruppierungen können zu einer Gesamtgruppierung verschmolzen werden.

Da der Algorithmus die Suche in lokalen Nachbarschaften erfordert, die Nachbarschaftsrelationen in den Polygondaten jedoch nicht direkt erkennbar sind (im Gegensatz zu äquidistant abgetasteten Bildpunktmatrizen), ermöglicht die Rückprojektion der Konturdaten auf ein Matrixraster eine schnelle Nachbarschaftssuche. Es soll hierbei von Relokalisation gesprochen werden. Besonders effizient läßt sich dies durch eine Matrix reduzierter Auflösung, die Reduced Resolution Link Matrix (RRLM) realisieren (vergleiche Bild 9). Die Konturen werden in der Farbe ihres Konturindex (daher der vertikale hell-dunkel Verlauf in Bild 9) und um den Reduktionsfaktor $r$ in der Auflösung reduziert, in die RRLM eingezeichnet. (Der Reduktionsfaktor $r$ ist der Quotient aus der Kantenlänge der ursprünglichen Bildpunktmatrix und der Kantenlänge der RRLM.) Durch die Auflösungsreduktion kann die Größe der zu durchsuchenden Nachbarschaften reduziert werden, was zu erheblichen Einsparungen an Rechenaufwand führt. Die Suche benachbarter Polygoneckpunkte ohne Relokalisation ist im Aufwand von der Ordnung $\mathcal{O}\left(n^{2}\right)$, wenn $n$ die Anzahl der Eckpunkte ist. Für den

Bild 8: Der Segmentieralgorithmus gruppiert Konturfragmente, die lokal benachbart sind.

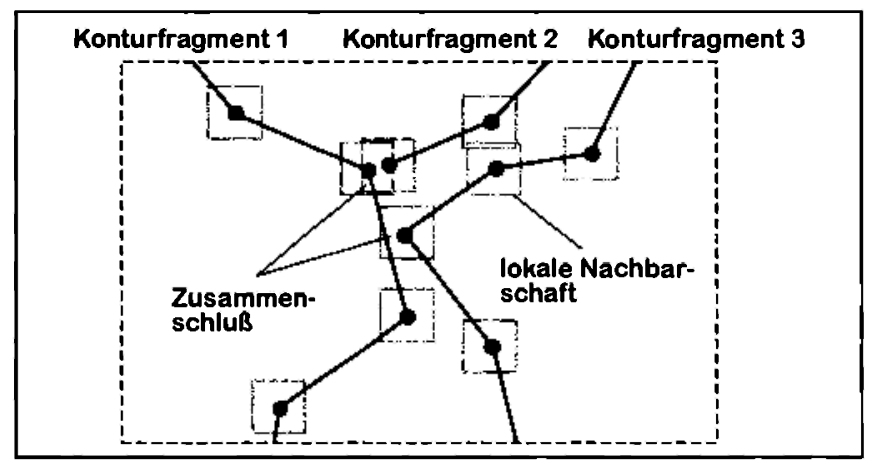




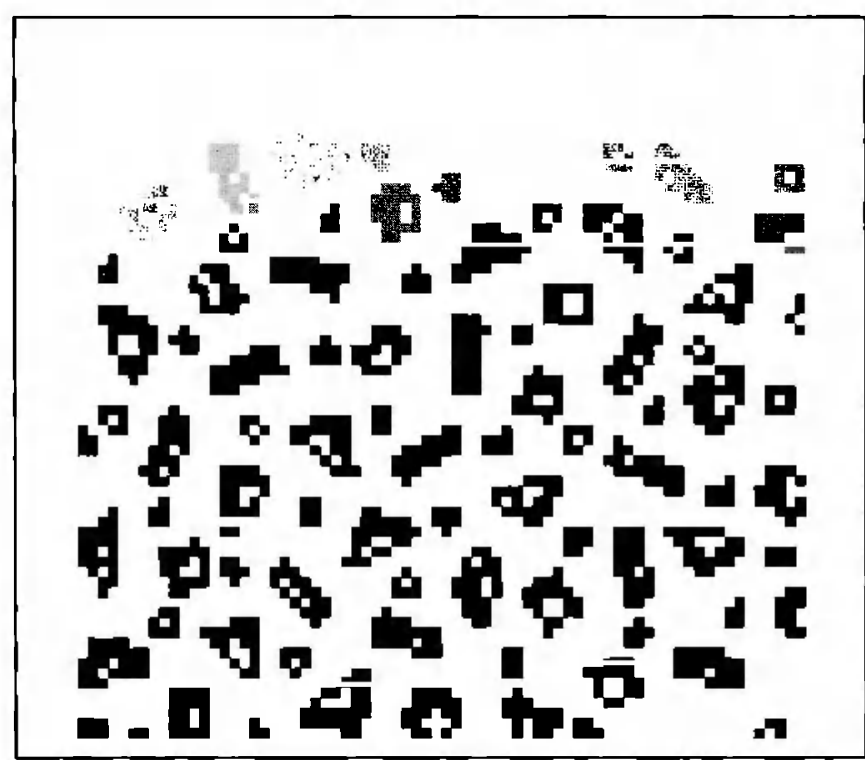

Bild 9: Beispiel einer Reduced Resolution Link Matrix zur Segmentierung der Konturobjekte aus Bild 3. (Reduktionsfaktor $r=7$.)

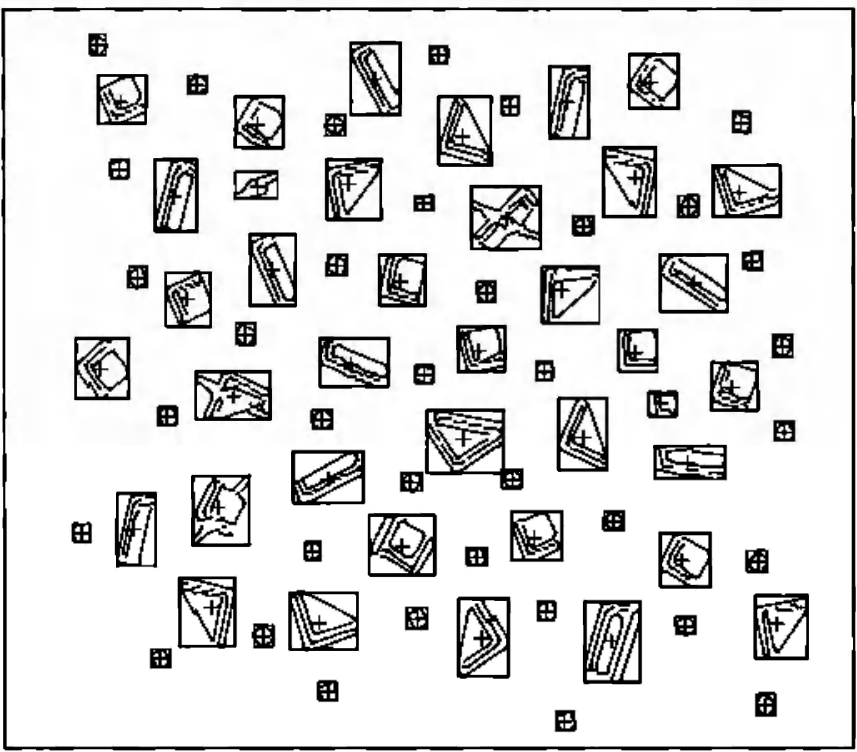

Bild 10: Segmentierte Texturelemente (74 Objekte).

vorgestellten Algorithmus ist die Nachbarschaftssuche von der Ordnung $\mathcal{O}\left(n \cdot\left(\frac{w}{r}\right)^{2}\right)$, wenn $w$ die Seitenlänge des Nachbarschaftsfensters in der Bildauflösung und $r$ den Reduktionsfaktor darstellt. Da $\left(\frac{w}{r}\right)^{2} \ll n$ gilt (für die hier betrachteten Beispiele gilt im allgemeinen $\left.\frac{w}{r} \approx 10^{-3} n\right)$, ist die Segmentierung entsprechend schnell.

Die Bilder 10 und 11 zeigen die segmentierten Konturobjekte zu den Beispielen aus Bild 3 und 6 . Wie im Vergleich zwischen den Bildern zu erkennen ist, werden an den Konturdaten keinerlei Manipulationen vorgenommen (außer der Entfernung der Randtexel

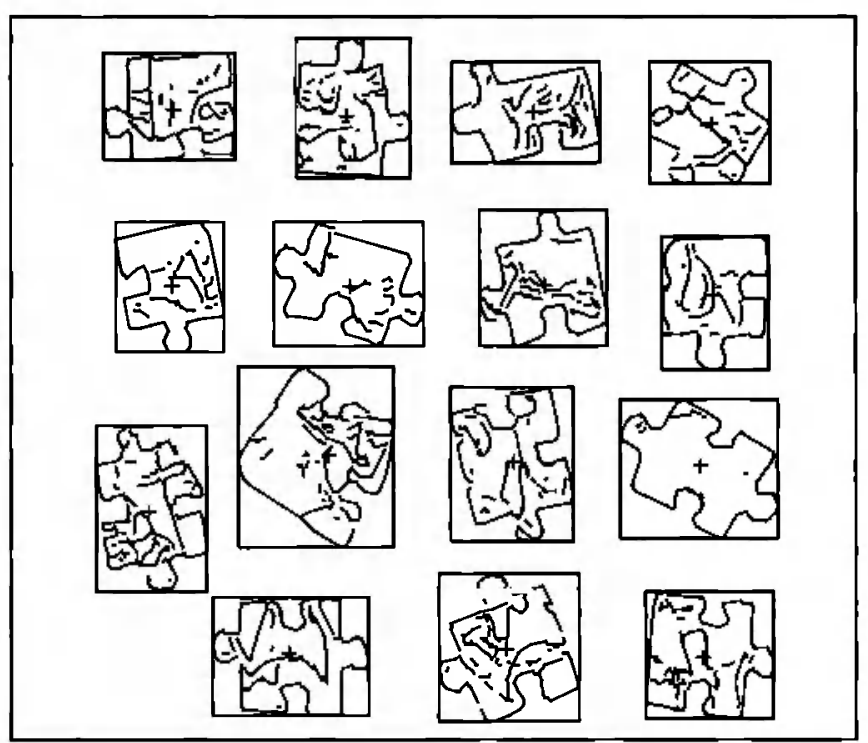

Bild 11: Segmentierte Puzzleteile (15 Objekte).

aus Bild 3). Die Konturfragmente werden lediglich logisch nach Objekten sortiert, was durch die rechtekkigen Rahmen angedeutet ist. Die Texelsegmentierung (Bild 10) ist für einen Reduktionsfaktor $r=7$ in $25 \mathrm{msec}$ auf einem mit $60 \mathrm{MHz}$ getakteten Pentium PC möglich. Für $r=1$ und entsprechend größerem Suchfenster $w=11$ dauert die Segmentierung $70 \mathrm{msec}$.

\section{Geometrische Linienmomente von Konturobjekten}

Bei der Beschreibung der grundlegenden Gleichungen des Linienmomentenansatzes wird von der Formulierung geometrischer Flächenmomente ausgegangen, die schon von [3] zur Objekterkennung herangezogen wurden und bis heute in zahlreichen Anwendungen und Modifikationen Forschungsgegenstand sind (siehe z.B. $[4 ; 5 ; 6 ; 7]$ ):

$$
m_{p q}^{(2)}=\iint_{(x, y) \in D(O)} x^{p} y^{q} f(x, y) \mathrm{d} A .
$$

$m_{p q}^{(2)}$ ist das geometrische Flächenmoment der Ordnung $(p, q)$ des Objekts $O$. Integriert wird dabei über den Definitionsbereich $D(O)$, der durch Segmentierung ermittelt wurde. $f(x, y)$ ist der Grauwert an der Stelle $(x, y)$. (Im Falle der Anwendung von Gl. (3) auf Bildmatrizen wird die Integration durch eine Summation ersetzt.) Nach dem sogenannten Uniqueness Theorem (vergleiche [3]) ist die Menge aller Momente $m_{p q}^{(2)}$ für $p, q=[0,1, \ldots, \infty[$ eineindeutig bestimmt durch Form und Aussehen des Objekts $O$ bzw. der Funktion $f(x, y)$.

Es soll nun von Flächenobjekten auf Konturobjekte übergegangen werden. Dazu ist das Flächenintegral durch ein Kurvenintegral zu ersetzen:

$$
m_{p q}^{(1)}=\int_{\mathcal{C}(O)} x(\bar{s})^{p} y(\vec{s})^{q} f(\bar{s}) \mathrm{d} \vec{s} .
$$


$m_{r}^{(1)}$ ist das geometrische Linienmoment der Ordnung $(p, q)$ des Konturobjekts $O$. Integriert wird entlang der Kontur $C(O)$, wobei $\vec{s}$ für den Bogen der Kontur steht. $f(\vec{s})$ wird lineare Dichte genannt und zum Zweck der reinen Formerkennung hier $f(\hat{s})=1$ gesetzt. Linienmomente nach Gl. (4) sind ebenso wie Flächenmomente schon lange bekannt. Im Gegensatz zu den Flächenmomenten hat ihre Anwendung in der Bildverarbeitung jedoch nach den Kenntnissen des Autors bisher nicht stattgefunden. (Linienmomente spielen vielmehr in der Mechanik als Trägheitsmomente von Kurven eine Rolle.) Zur Berechnung der Linienmomente nach Gl. (4) ist die vektorielle Formulierung nach den kartesischen Koordinaten $x$ oder $y$ aufzulösen. Dazu kann der Betrag des Bogenelements folgendermaßen ausgedrückt werden:

$$
\mathrm{d} s=\sqrt{1+\left(y^{\prime}(x)\right)^{2}} \mathrm{~d} x=\sqrt{1+\left(x^{\prime}(y)\right)^{2}} \mathrm{~d} y .
$$

Die Linienmomente $m_{p q}^{(1)}$ lassen sich nun wie folgt berechnen:

$$
\begin{aligned}
m_{p q}^{(1)} & =\int_{C(O)} x^{p} y(x)^{q} \sqrt{1+\left(y^{\prime}(x)\right)^{2}} \mathrm{~d} x= \\
& =\int_{C(O)} x(y)^{p} y^{q} \sqrt{1+\left(x^{\prime}(y)\right)^{2}} \mathrm{~d} y .
\end{aligned}
$$

Wenn die Integration über $x$ erfolgen soll, sind die Werte $y(x)$ auf der Kontur $C(O)$ in Abhängigkeit von $x$ zu parametrieren. Bei Integration über $y$ ist entsprechend $x(y)$ in $y$ auszudrücken.

\subsection{Effiziente Berechnung geometrischer \\ Linienmomente}

Die im letzten Abschnitt vorgestellten Formulierungen von geometrischen Linienmomenten sind für beliebige Kurventypen gültig. Durch die Additivität der Integration nach Gl. (4) kann die Kontur $C(O)$ beliebig oft unterbrochen sein. Im folgenden soll die Gleichung (6) für den Fall polygonaler Kurven gelöst werden.

Gemäß der Definition von Konturobjekten nach Gl. (2) ergeben sich die Linienmomente $m_{p q}^{(1)}$ aus der Summe der Beiträge einzelner Geradenstúcke $c_{\imath}$. (Da die Konturfragmentierung bei der Betrachtung einzelner Geradenstücke keine Rolle mehr spielt, wird im folgenden der Index $j$ weggelassen.)

$$
m_{p q}^{(1)}=\sum_{i} \int_{c_{i}} x(\vec{s})^{p} y(\vec{s})^{q} f(\vec{s}) d \vec{s}=\sum_{i} D_{i} .
$$

$D_{i}$ ist also der Beitrag des Geradenstücks $i$ zum Gesamtmoment $m_{\rho_{4}}^{(1)}$ des betrachteten Konturobjekts. Führen wir die Berechnung durch Integration über $x$ aus (Gl. (6), 1. Zeile), dann können die Geradensegmente wie folgt parametriert werden:

$c_{i}: y=a_{i} x+y_{i}-a_{i} x_{i}, \quad x_{i} \leq x<x_{i+1}, \quad i=1,2, \ldots, n$.

Das Konturobjekt bestünde aus $n$ Geradensegmenten, $a_{i}$ ist die Steigung des $i$-ten Segments. (Bei nahezu

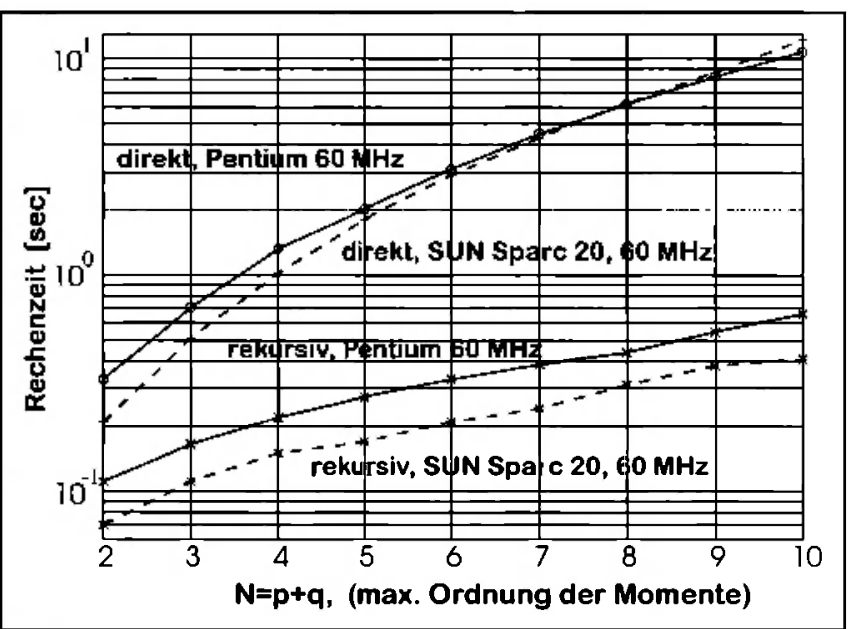

Bild 12: Rechenzeiten zur Bestimmung aller Linienmomente der Ordnung $(p+q) \leq N$ für das Beispiel aus Bild 3 mit 2789 Polygoneckpunkten.

vertikalen Geradenstücken ist die Integration über $y$ aus numerischen Gründen durchzuführen, vergleiche [1].) Es ergibt sich als Lösung

$$
\begin{aligned}
D_{i} & =\int_{x_{i}}^{x_{i+1}} x^{p}\left(a_{i} x+y_{i}-a_{i} x_{i}\right)^{q} \sqrt{1+a_{i}^{2}} \mathrm{~d} x \\
& =\sqrt{1+a_{i}^{2}} \sum_{k=0}^{q}\left(\begin{array}{l}
q \\
k
\end{array}\right) a_{i}^{k}\left(y_{i}-a_{i} x_{i}\right)^{q-\dot{k}} \frac{r_{i+1}^{p+k+1}-x_{i}^{p+k+1}}{p+k+1}
\end{aligned}
$$

Die Polygondaten (Polygoneckpunkte $\left(x_{i}, v_{i}\right)$ ) können also in der Form, wie sie nach der Echtzeitvektorisierung und der logischen Sortierung durch die Segmentierung angefallen sind, direkt weiterverarbeitet werden. Es läßt sich zusätzlich eine erhebliche Einsparung an Rechenaufwand durch den in [1] vorgestellten, rekursiven Algorithmus anstelle der direkten Lösung nach Gl. (9) erzielen.

Einen Vergleich der Rechenzeiten für die direkte Lösung nach Gl. (9) und die rekursive Lösung nach [1] zeigt Bild 12. Es wurden jeweils alle Linienmomente bis zu einer Ordnung $(p+q) \leq N$ für das Beispiel aus Bild 3 mit 2789 Polygoneckpunkten berechnet. Man erkennt deutlich den Geschwindigkeitsvorteil der rekursiven Lösung. Für die in Abschnitt 6 vorgestellen Ergebnisse wurden Merkmale aus Linienmomenten bis zur Ordnung $N=7$ herangezogen. In diesem Fall liegen die Rechenzeiten zur Analyse der gesamten Szene bei ca. $250 \mathrm{msec}$ (vergleiche Bild 12) bei einem mit $60 \mathrm{MHz}$ getakteten Prozesssor. Für aktuelle, handelsübliche Prozesssorgenerationen ist eine Beschleunigung um den Faktor $3-4$ anzusetzen.

\section{4 Übertragung des Linienmomenten- konzepts auf andere Momententypen}

Neben den geometrischen Flächenmomenten wurden in der Literatur zahlreiche weitere Momententypen definiert und vorgeschlagen, mit dem Ziel, bessere Merkmale in Anwendungen zur Objekterkennung zu erhalten. So basieren z.B. die Zernike Momente ([8]), 


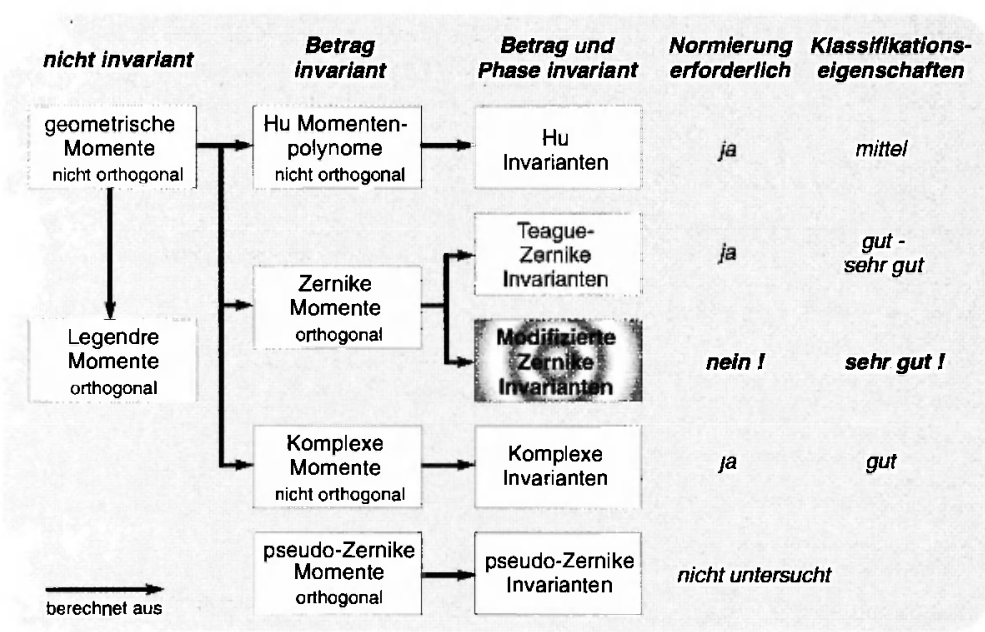

Bild 13: Invarianzeigenschaften bezüglich Rotation, Orthogonalität der Basisfunktionen und Überführbarkeit der betrachteten Linienmomententypen.

die Pseudo Zernike Momente (z.B. [4]) und die Legendre Momente (z.B. [4]) auf orthogonalen Basisfunktionen im Integranden der Momentendefinition, was zu unabhängigen Merkmalen führt. Im Vorgriff auf die Berechnung rotatorischer Invarianten in Abschnitt 5 ist außerdem zu bemerken, daß bestimmte Momententypen, die im allgemeinen komplexe Zahlen bilden, bereits im Betrag invariant gegenüber der Verdrehung von Objekten sind. Dies gilt für Zernike Momente, Pseudo Zernike Momente und Komplexe Momente ([9]). Die meisten Momententypen sind dabei ineinander überführbar. Die Invarianzeigenschaften, Orthogonalität der Basisfunktionen und Überführbarkeit der hier weiter betrachteten Momententypen ist in Bild 13 dargestellt.

Analog zu der Übertragung des Flächenmomentenansatzes auf Konturintegrale, wie in den Gleichungen (3) und (4) dargestellt, läßt sich das Linienmomentenkonzept auf die große Anzahl der aus der Literatur bekannten Momententypen anwenden. Damit eröffnen sich dem Ansatz eine Vielzahl bestehender Theorien bei entsprechender Anpassung der Formalismen. In Bezug auf die Berechnung von Invarianten in Abschnitt 5 werden im folgenden zwei neue Linienmomententypen vorgestellt.

Die Zernike Linienmomente $A_{n L}^{(1)}$ ergeben sich in Analogie zu den Zernike Flächenmomenten $A_{n L}^{(2)}$ (siehe [8]) $\mathrm{zu}$

$$
i_{n L}^{(1)}=\frac{n+1}{\pi} \int_{C(O)}\left[R_{n L}(\bar{s})\right] e^{-I L \phi(\vec{s})} f(\vec{s}) \mathrm{d} \vec{s}
$$

mit $n \in \mathrm{IN}, n \geq 0, n \geq L \geq 0$ und $(n-L)$ gerade. Die Ordnung der Momente $A_{:: L}^{(i)}$ ist $n, R_{n L}$ ist ein Polynom in der polaren Koordinate $r$ (Radius) und ist wie folgt definiert (vergleiche [5]):

$$
R_{n L}(r(\bar{s}))=\sum_{\substack{k=L \\ n-k \text { even }}}^{n} B_{n L k} r(\bar{s})^{k}
$$

$$
\text { mit } B_{n L k}=\frac{(-1)^{\frac{n-k}{2}}\left(\frac{n+k}{2}\right) !}{\left(\frac{n-k}{2}\right) !\left(\frac{L+k}{2}\right) !\left(\frac{k-L}{2}\right) !} \text {. }
$$

Die Zernike Linienmomente lassen sich aus den geometrischen Linienmomenten berechnen:

$$
\begin{aligned}
A_{n L}^{(1)}= & \frac{n+1}{\pi} \sum_{\substack{k=L \\
n-k \text { even }}}^{n} \sum_{m=0}^{L} \sum_{j=0}^{\frac{k-L}{7}} B_{n L k}(-i)^{n z}\left(\begin{array}{c}
L \\
m
\end{array}\right) \\
& \left(\begin{array}{c}
\frac{k-L}{2} \\
j
\end{array}\right) m_{(k-m-2 j),(m+2 j)}^{(1)} .
\end{aligned}
$$

Aus der Definition der Zernike Linienmomente nach Gl. (10) in polaren Koordinaten $(r, \phi)$ folgt sofort, daß $\left|i_{n L}^{(1)}\right|$ invariant gegenüber Objektdrehungen ist.

Des weiteren sollen noch die Komplexen Linienmomente $C_{p q}^{(1)}$ in Analogie zu den Komplexen Flächenmomenten $C_{p q}^{(2)}([9 ; 10])$ definiert werden. Sie ergeben sich zu:

$$
\begin{aligned}
C_{p q}^{(1)} & =\int_{C(O)}(x(\vec{s})+i y(\bar{s}))^{p}(x(\vec{s})-i y(\vec{s}))^{q} f(\vec{s}) \mathrm{d} \vec{s} \\
& =\int_{C(O)} r(\vec{s})^{p+q} e^{i(p-q) \phi(\vec{s})} f(\vec{s}) \mathrm{d} \vec{s} .
\end{aligned}
$$

Auch für die Komplexen Linienmomente gilt, daß $\left|C_{p q}^{(1)}\right|$ bereits invariant gegenüber Objektverdrehungen ist. Die Komplexen Linienmomente stehen in folgendem Zusammenhang zu den geometrischen Linienmomenten:

$C_{p q}^{(1)}=\sum_{r=0}^{\bar{p}} \sum_{s=0}^{\bar{q}}\left(\begin{array}{l}p \\ r\end{array}\right)\left(\begin{array}{l}q \\ s\end{array}\right) e^{p+q-(r+s)}(-1)^{q-s} m_{(r+s),(p+q-(r+s))}^{(1)}$

Die Definition von Legendre Linienmomenten und Pseudo Zernike Linienmomenten (vergleiche Bild 13) erfolgt analog zu obigem Vorgehen.

\section{Invarianten}

Nachdem die grundlegenden Beziehungen zur Berechnung verschiedener Linienmomententypen vorgeführt wurden, sind für die praktische Anwendung der Linienmomente zur Texturelement- oder Objekterkennung bestimmte Forderungen zu stellen. So sollen die Merkmale, die zur Klassifikation und Fehlererkennung herangezogen werden, im allgemeinen unabhängig von der Lage der Objekte im Bild sein (translatorische Invarianz). Vielfach ist auch gefordert, $\mathrm{da} ß$ die Merkmale der Objekte unabhängig von deren Größe im Bild bzw. der Kameraentfernung zum Objekt 
sind (Skalierungsinvarianz). Außerdem kann die Unabhängigkeit von der Winkellage der Objekte erwünscht sein, man spricht dann von rotatorischer Invarianz.

Im folgenden soll dargestellt werden, wie die Gleichungen der Linienmomente zu modifizieren sind bzw. wie durch Kombination von Linienmomenten verschiedener Ordnung die gewünschten Invarianzeigenschaften erzielt werden können. Die entsprechenden Umformungen sind natürlich nur dann durchzuführen, wenn die korrespondierende Invarianzeigenschaft tatsächlich gefordert ist.

Translatorische Invarianz kann für jedes Objekt erreicht werden, indem von den Koordinaten $(x, y)$ im Integranden jeweils die Linienschwerpunktskoordinate $(x, y)$ abgezogen wird:

$$
\xi_{p q}^{(1)}=\int_{C(O)}(x(\vec{s})-x)^{p}(y(\vec{s})-\bar{y})^{q} f(\vec{s}) \mathrm{d} s .
$$

$\xi_{p q}^{(1)}$ ist dann das zentrale Linienmoment der Ordnung $(p+q)$. Die schnelle Berechnung der zentralen Linienmomente kann wie in Abschnitt 3.1 erläutert durchgeführt werden, wenn im voraus alle Koordinaten $x$ und $y$ auf den Linienschwerpunkt bezogen werden.

Zur Erzielung von Skalierungsinvarianz sind die Koordinaten $(x, y)$ sowie die Integrationsvariable $\vec{s}$ auf die Länge der Konturen $l_{C}$ des Konturobjekts $C(O)$ zu beziehen

$\mu_{p q}^{(l)}=\int_{C(o)}\left(\frac{x(\vec{s})-\bar{x}}{l_{C}}\right)^{p}\left(\frac{y(\vec{s})-\bar{y}}{l_{C}}\right)^{q} \frac{\mathrm{d} \vec{s}}{l_{C}}=\frac{\xi_{p q}^{(1)}}{\left(\xi_{00}^{(1)}\right)^{\gamma_{1}}}$

mit $\gamma_{1}=p+q+1$. Die skalierungsinvarianten, zentralen Linienmomente $\mu_{p q}^{(1)}$ sind also über den Quotienten aus zentralen Linienmomenten berechenbar $\left(\xi_{00}^{(1)}=l_{C}\right.$ ist die Gesamtlänge der Konturen). Der Exponent $\gamma_{1}$ weicht von dem entsprechenden Exponenten zur Erzielung von Skalierungsinvarianz bei Flächenmomenten $a b$. Eine andere, weniger rauschempfindliche Möglichkeit zur Bildung skalierungsinvarianter Merkmale entsteht durch das Beziehen der Koordinaten auf das sogenannte polare Linienträgheitsmoment $I_{p}=\xi_{20}^{(1)}+\xi_{02}^{(1)}$. Dieses hat die Dimension einer Länge zur Potenz 3, womit sich folgende Normierung ergibt

$\psi_{p q}^{(1)}=\int_{C(O)}\left(\frac{(x(\bar{s})-\bar{x})^{3}}{I_{p}}\right)^{\frac{p}{3}}\left(\frac{(y(\bar{s})-\bar{y})^{3}}{I_{p}}\right)^{\frac{q}{3}} \frac{\mathrm{d} \vec{s}}{I_{p}^{\frac{1}{3}}}=\frac{\xi_{p q}^{(1)}}{\left(\xi_{20}^{(1)}+\xi_{02}^{(I)}\right)^{\gamma_{2}}}$

mit $\gamma_{2}=\frac{p+c_{1}+1}{3}$. Auch hier weicht der Exponent $\gamma_{2}$ von dem entsprechenden Ansatz bei Flächenmomenten (siehe z.B. [5]) ab.

Es soll nun auf die Bildung der rotatorischen Invarianten eingegangen werden, die in Abschnitt 6 weiter ausgewertet werden. Auf die vollständige Darstellung der Berechnungsvorschriften wird verzichtet, da diese von den Gleichungen für Flächenmomente
Tabelle 1: Untersuchte Typen, Ordnung $N$ sowie Anzahl von Linienmomenten-Invarianten (LMI).

\begin{tabular}{|l|l|l|l|}
\hline Typ & Abkürzung & $N$ & Anzahl \\
\hline Hu LMI, [3] & HLMI & 7 & 32 \\
\hline Teague-Zernike LMI, [8] & TZLMI & 7 & 32 \\
\hline $\begin{array}{l}\text { Modifizierte Zernike LMI, } \\
\text { Eigenentwicklung }\end{array}$ & MZLMI & 7 & 32 \\
\hline Komplexe LMI, [9] & CLMI & 7 & 78 \\
\hline Traditionelle Hu LMI, [3] & THLMI & 3 & 7 \\
\hline
\end{tabular}

kaum abweichen. Bild 13 veranschaulicht den Zusammenhang zwischen Linienmomententypen und den aus ihnen berechneten rotatorischen Invarianten.

In Tabelle 1 sind die in diesem Artikel untersuchten Invariantengruppen mit Bezeichnung und Abkürzung sowie einer einschlägigen Literaturreferenz aufgeführt. Außerdem ist die maximale Ordnung der Linienmomente $N$ mit $(p+q) \leq N$ gegeben, aus denen der Invariantensatz berechnet wird, dessen Größe in der rechten Spalte angegeben ist.

Schon $H u$ hat in [3] die Berechnung algebraischer Invarianten aus geometrischen Flächenmomenten vorgeführt. Dazu werden zuerst komplexe Momentenpolynome gebildet, die im Betrag bereits invariant gegenüber Objektverdrehungen sind (vergleiche Bild 13). Die Hu Linienmomentenpolynome berechnen sich aus den geometrischen Linienmomenten zu

$I_{p-r, r}^{(1)}=\sum_{l=0}^{p-2 r}(-i)^{l}\left(\begin{array}{c}p-2 r \\ \vdots\end{array}\right) \sum_{k=0}^{r}\left(\begin{array}{l}r \\ l\end{array}\right) m_{(p-2 k-i) \cdot(2 k+i)}^{(1)}$

mit $p-2 r>0$ (vergleiche $[3 ; 5])$. Sie erreichen damit einen Invarianzstatus, der den Zernike Linienmomenten, den Pseudo Zernike Linienmomenten und den Komplexen Linienmomenten bereits innewohnt (siehe Bild 13). Durch nichtlineare Kombination der Momentenpolynome entstehen reelle Invarianten, die als Merkmale zur Klassifikation herangezogen werden können. In diesem Aufsatz werden zwei Gruppen von Hu-Invarianten unterschieden. Zum einen ein 32 Invarianten zählender Merkmalssatz, der auf Momenten bis zur Ordnung 7 basiert (HLMI, Tabelle 1) und zum anderen die 7 traditionellen Hu LinienmomentenInvarianten THLMI, die auf Momenten bis zur Ordnung 3 basieren. Die THLMI wurden gesondert aufgeführt, da die entsprechenden FlächenmomentenInvarianten in der Literatur oft zitiert werden.

Die Teague-Zemike Linienmomenten-Invarianten (TZLMI) basieren auf den Zernike Linienmomenten nach Gl. (10). Durch Betragsbildung und nichtlinearer Kombination der Momente entstehen reelle Invarianten ([8]).

Die Modifizierten Zernike Linienmomenten-Invarianten (MZLMI) basieren ebenfalls auf den Zernike Linienmomenten nach Gl. (10) und stellen als Abwandlung der in [5] vorgeschlagenen Invarianten eine Eigenentwicklung dar. Die Invariantenbildung basiert dabei auf folgendem Ansatz 


$$
(\mathrm{MZLMI})_{n, n+z}=\left[A_{m h}^{(1) *}\left(A_{n L}^{(1)}\right)^{p}\right] \pm\left[A_{m h}^{(1) *}\left(A_{n L}^{(1)}\right)^{p}\right]^{*}
$$

mit $\mathrm{h} \leq L, m \leq n, p=h / L$ und $z=L / h .\left((\cdot)^{*}\right.$ kennzeichnet das konjugiert komplexe Element.) Durch $0 \leq p \leq 1$ wird erreicht, daß die zahlenmäßigen Größenordnungen von Invarianten, basierend auf Momenten verschiedener Ordnung $n$, gleich sind. Dies wirkt sich vorteilhaft bei der Klassifikation aus, eine besondere Normierung der Invarianten ist damit nicht erforderlich (vergleiche Bild 13 und Abschnitt 5.1).

Die komplexen Linienmomenten-Invarianten (CLMI) basieren auf den Komplexen Linienmomenten nach Gl. (13). Sie entstehen durch einen Ansatz der Form

$(\mathrm{CLMI})_{r s k}=\left[C_{r s}^{(1)}\left(C_{t u}^{(1)}\right)^{k}\right]+\left[C_{r s}^{(1)}\left(C_{t u}^{(1)}\right)^{k}\right]^{*}$

mit $(r-s)+k(t-u)=0$ (vergleiche [9]).

\subsection{Normierung der Invarianten}

Für alle Linienmomente gilt, daß mit zunehmender Ordnung der Momente die Größenordnung ihrer Zahlenwerte stark zunimmt. Zur Klassifikation vektorieller Merkmale ist es dagegen anzustreben, daß die Größenordnungen jeder Merkmalsdimension etwa gleich sind. Zur Erzielung hoher Klassifikationsgüten ist daher eine Normierung der Momente bzw. der Invarianten erforderlich. Vorstellbar wäre hier z.B. die $(\mu, \sigma)=(0,1)$ Normierung in allen Merkmalsdimensionen. Es zeigt sich jedoch, daß auf diese Weise Unterschiede in den Invarianten, die durch unterschiedliche Objektformen begründet sind, unkenntlich gemacht werden. Als bessere Methode hat sich daher erwiesen, die Linienmomente oder abgeleitete Größen entsprechend ihrer Ordnung zu normieren. Folgende Normierungen werden vorgeschlagen:

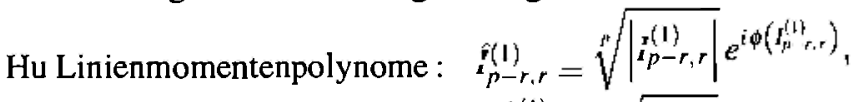
Zernike Linienmomente : $\quad \hat{A}_{n L}^{(1)}=\sqrt[n]{\left|A_{n L}^{(1)}\right|} e^{i \phi\left(A_{n L}^{(1)}\right)}$.

Für die Komplexen Linienmomente hat sich die folgende Normierung der Invarianten bewährt:

CLMI : $(\text { CLMI })_{r s k}^{\wedge}=\operatorname{sgn}\left((\text { CLMI })_{r s k}\right) \cdot \sqrt[k+1]{\mid(\text { CLMI })_{r s k} \mid}$.

\section{Anwendungen}

In diesem Abschnitt werden die Ergebnisse zweier Anwendungen des Linienmomentenansatzes vorgestellt. Es soll dabei zuerst auf die prinzipielle Vorgehensweise zur Merkmalsauswahl und Klassifikation eingegangen werden.

In Tabelle 1 sind die untersuchten Invariantengruppen aufgeführt. Es fällt auf, daß̉ bei der Berechnung der Invarianten aus Momenten bis zur Ordnung 7, die Anzahl der Merkmale für ein Objekt sehr hoch ist (rechte Spalte in Tabelle 1). Da bei zu hoher Merkmalsdimen- sion die Erkennungsleistung von Klassifikatoren im allgemeinen abnimmt, sind Verfahren der Merkmalsselektion einzusetzen. Für niederdimensionale Merkmale sind außerdem die Klassifikationszeiten klein.

Für die vorliegenden Untersuchungen wurde ein selbstentwickelter, nichtparametrischer, auf dem KNächste-Nachbarn (KNN) Prinzip beruhender Gütewert zur Beurteilung von Merkmalsverteilungen im Merkmalsraum berechnet. Ein heuristisches Suchverfahren (sequentielle Rückwärtsselektion, z.B. [11]) und Genetische Algorithmen wurden zur Durchsuchung der Merkmalskombinationen eingesetzt. Zur Definition des Gütewertes und Erläuterungen zur Merkmalsselektion sei auf [12] verwiesen. Im Rahmen der angestellten Untersuchungen wurde die Erkennungsleistung der Klassifikation immer für den vollen Merkmalssatz (full set), den über Merkmalsselektion bestimmten besten Merkmalssatz (best set) und den auf 7 Merkmale reduzierten besten Merkmalssatz (reduced set) bestimmt. Letzterer wurde untersucht, um einen Vergleich mit dem 7-dimensionalen Merkmalssatz der THLMI zu ermöglichen.

Zur Klassifikation wurde ein Maximum-LikelihoodNormalverteilungsklassifikator mit Rückweisung verwendet. Die Annahme normalverteilter Merkmale ist hier gerechtfertigt, da die Merkmale gerade invariant gegenüber den betrachteten Transformationen (Translation, Skalierung und Rotation) sind. Lediglich das Rauschen in den Objektformen und der Vektorisierung wird zu einer Streuung der Merkmale führen, für die die Annahme einer Normalverteilung zutrifft (siehe Betrachtungen in [12]). Die Entscheidung über Klassenzugehörigkeiten bzw. Rückweisungen fehlerhafter Texturelemente und Objekte erfolgt über den Mahalanobisabstand $d_{M}(\boldsymbol{m}, \omega)$ der Merkmalsvektoren $\boldsymbol{m}$ zur Klasse $\omega \in \Omega=\left[\omega_{1}, \ldots, \omega_{K}\right]$

$$
d_{M}^{2}(\boldsymbol{m}, \omega)=\left(\boldsymbol{m}-\boldsymbol{\mu}_{\omega}\right)^{T} \cdot C_{\omega}^{-1} \cdot\left(\boldsymbol{m}-\boldsymbol{\mu}_{\omega}\right)
$$

mit $\boldsymbol{\mu}_{\omega}$ und $C_{\omega}$ als dem klassenspezifischen Zentrumsvektor und der Klassen-Kovarianzmatrix.

Die Klassifikation erfolgt nun nach folgender Entscheidung:

$$
\begin{aligned}
\boldsymbol{m} \in \omega_{k}: d_{M}\left(\boldsymbol{m}, \omega_{k}\right)= & \min _{\omega_{k}}\left\{d_{M}\left(\boldsymbol{m}, \omega_{k}\right)\right\} \wedge \\
& d_{M}\left(\boldsymbol{m}, \omega_{k}\right)<d_{M, \text { grenz }},
\end{aligned}
$$

$m \notin \Omega$ : sonst.

Die Erkennung eines fehlerhaften Objekts erfolgt also nicht durch die Modellierung einer eigenen Fehlerklasse, da hierzu im allgemeinen nicht genug Fehlerwissen vorliegt. Vielmehr wird ein Fehler durch große Mahalanobisabstände zu den bekannten Objektklassen $\Omega$ detektiert. Sind die Mahalanobisabstände eines Merkmalsvektors zu allen bekannten Klassen $\Omega$

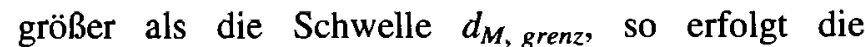
Rückweisung, d.h. die Detektion eines Fehlers.

Zur quantitativen Beurteilung der Klassifikationsgüten werden nun zwei Maßzahlen definiert. Die Klassifikationsgiite $q$ eines Testdatensatzes aus $M$ Vektoren ist wie folgt definiert (anstelle der Klassen 
$\omega_{k}$ werden hier Klassenindizes $k=[1, \ldots, K]$ verwendet):

$$
\begin{aligned}
d_{M}\left(\boldsymbol{m}, k^{*}\right) & =\min _{k}\left\{d_{M}(\boldsymbol{m}, k)\right\}, \\
q_{m}^{*} & =\frac{1}{K-1} \sum_{\substack{k=1 \\
k \neq k^{*}}}^{K}\left(1-\frac{d_{M}\left(\boldsymbol{m}, k^{*}\right)}{d_{M}(\boldsymbol{m}, k)}\right), \\
q_{m} & =\left\{\begin{array}{l}
+q_{m}^{*}, \omega_{k^{*}}=\omega_{\text {soll }} \\
-q_{m}^{*}, \omega_{k^{*}} \neq \omega_{\text {soll }}
\end{array}, \quad q=\frac{1}{M} \sum_{m=1}^{M} q_{m} .\right.
\end{aligned}
$$

$q$ ergibt sich also als Mittelwert aus Einzelgüten $q_{m}$, die für jeden Merkmalsvektor Werte zwischen -1 und +1 annehmen können. Positive $q_{m}$ liegen vor, wenn die Entscheidung des Klassifikators richtig war $\left(\omega_{k^{*}}=\omega_{\text {soll }}\right)$, negative, wenn die Entscheidung falsch war. Werte $q_{m} \bar{\sim}+1$ stehen dabei für eine sichere, richtige Entscheidung, $q_{m} \approx 0$ für eine unsichere Entscheidung und $q_{m} \approx-1$ für eine sichere, falsche Entscheidung des Klassifikators.

Die Rückweisungssicherheit $\Delta$ für fehlerhafte Objekte soll in Worten definiert werden:

$d_{M . R_{\min }}:$ minimale Mahalanobisdistanz aller Sollrückweisungen zu allen Klassen,

$d_{M . G_{\max }}:$ maximale Mahalanobisdistanz aller "Gut"Objekte zu ihren Sollklassen,

womit $\Delta=d_{M, R_{\min }}-d_{M, G_{\max }}$ definiert wird. Sowohl zur Berechnung der Klassifikationsgüte $q$ wie für die Rückweisungssicherheit $\Delta$ ist die Kenntnis der Sollklassen und Sollrückweisungen des Testdatensatzes erforderlich. Die Klassifikationsgüte $q$ macht dabei keine Aussage über die Erkennbarkeit fehlerhafter Objekte. Die Rückweisungssicherheit $\Delta$ dagegen kennzeichnet exakt die Intervallbreite, in der die Schwelle $\mathrm{d}_{M, \text { grenz }}$ liegen darf, so daß die erwünschte Sollklassifikation für den Testdatensatz stattfindet. Sie macht also direkt eine Aussage über die Robustheit, mit der die fehlerhaften Elemente im Testdatensatz erkannt werden. Es sollte also $\Delta$ möglichst groß und positiv sein. Für $\Delta<0$ ist der Testdatensatz für kein $d_{M, \text { grenz }}$ fehlerfrei klassifizierbar. Das $\mathrm{Ma} B \Delta$ ist kein statistischer Mittelwert (im Gegensatz zu q) sondern ein Extremwert, der für das am schwierigsten als solches zu erkennende, fehlerhafte Objekt gilt.

\subsection{Inspektion von Texturen aus Texeln}

Als erste Anwendung soll die Folieninspektionsaufgabe betrachtet werden, bei der die Folienoberfläche als strukturierte Textur aus Texeln aufgefaßt werden kann. Die Grauwertszene, das Konturbild und die segmentierten Texel einer Beispielaufnahme mit 8 fehlerhaften Texturelementen ist in den Bildern 2, 3 und 10 dargestellt. Die Trainingsdaten zur Berechnung der klassenspezifischen Normalverteilungen wurden aus 10 unabhängigen Bildern mit jeweils

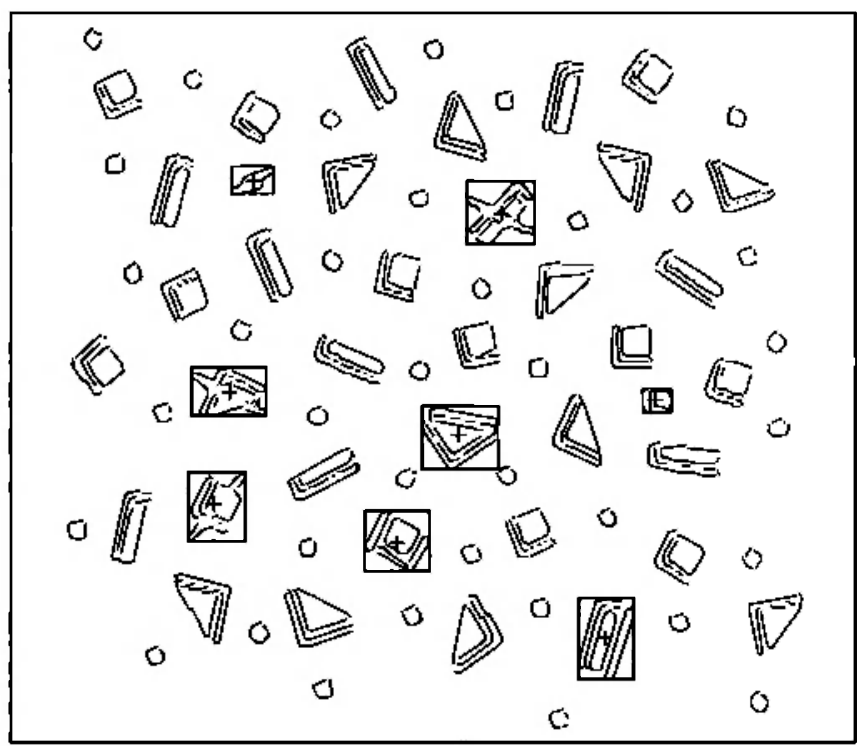

Bild 14: Erfolgreich erkannte, fehlerhafte Texturelemente.

ca. 75 fehlerfreien Texeln (767 Trainingsvektoren) aus den 4 Texelklassen (Rechtecke, Dreiecke, Quadrate, kleine Quadrate, vergl. Bild 2) gewonnen. Getestet wurde an 4 Bildern mit jeweils ca. 75 Texeln (296 Testvektoren) aus den 4 Klassen und jeweils 8 fehlerhaften Texturelementen (siehe Bild 2).

Bild 14 zeigt die in einem Testbild erfolgreich erkannten, fehlerhaften Texel, die durch einen Rahmen gekennzeichnet sind. Zur qualitativen Veranschaulichung der hohen Signifikanz der Merkmale, ist in Bild 15 die Merkmalsverteilung des gesamten Testdatensatzes visualisiert. Es handelt sich hierbei um das 6 Merkmale zählende best set der TZLMI (vergleiche Tabelle 2). Zur Projektion des 6-dimensionalen Merkmalsraumes auf 3 Dimensionen wurde der Sammons nonlinear mapping Algorithmus nach [13] eingesetzt. Man erkennt deutlich die gute Clusterbildung für die 4 zu unterscheidenden Texelklassen. Alle fehlerhaften Texel (schwarze Punkte in Bild 15) weichen eindeutig von den Klassenclustern ab.

Bild 15: Visualisierung der Merkmalsverteilung des Testdatensatzes (Texturbeispiel), Projektion von 6 auf 3 Dimensionen (Sammons Algorithmus).

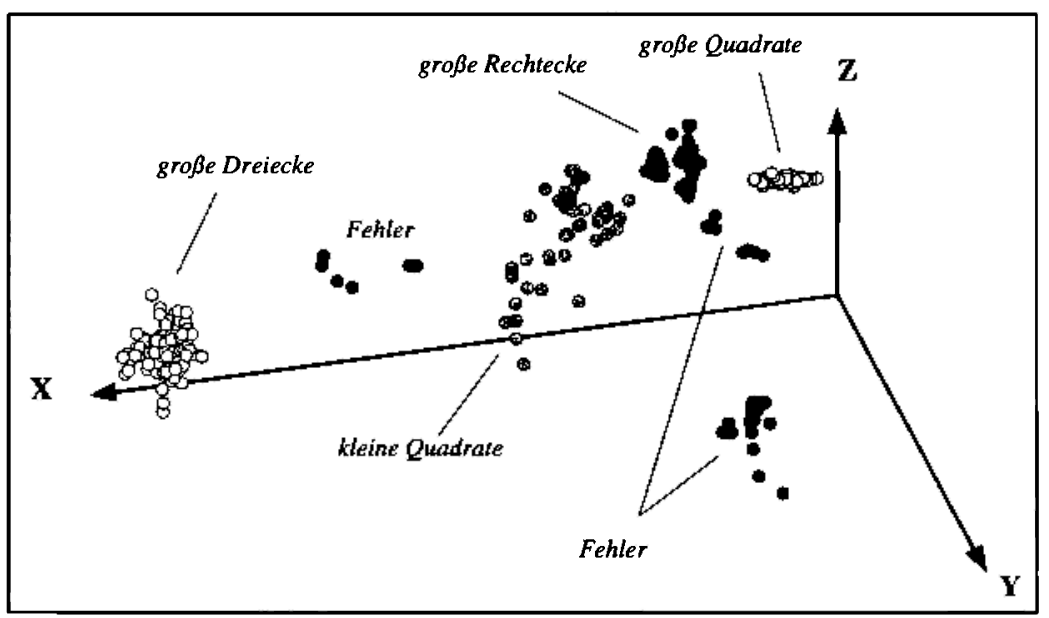


Tabelle 2: Merkmalsdimension $D$, Klassifikationsgüte $q$, Rückweisungssicherheit $\Delta$ und Klassifikationsfehler $e$ für die untersuchten Invarianten und Merkmalssätze des Testdatensatzes der Textur aus Texeln.

\begin{tabular}{|c|c|c|c|c|c|c|c|c|c|c|c|c|c|}
\hline \multirow[t]{2}{*}{ Invarianten } & \multirow[b]{2}{*}{$N$} & \multicolumn{4}{|c|}{ full set } & \multicolumn{4}{|c|}{ best set } & \multicolumn{4}{|c|}{ reduced set } \\
\hline & & $D$ & $q$ & $\Delta$ & $e[\%]$ & $D$ & $q$ & $\Delta$ & $e[\%]$ & $D$ & $q$ & $\Delta$ & $e[\%]$ \\
\hline HLMI & 7 & 32 & 0,954 & 12,6 & 0 & 23 & 0,931 & 4,0 & 0 & 7 & 0,673 & $-6,7$ & 19,9 \\
\hline TZLMI & 7 & 32 & 0,993 & 36,2 & 0 & 6 & 0,974 & 7,2 & 0 & 7 & 0,974 & 7,5 & 0 \\
\hline MZLMI & 7 & 32 & 0,976 & 43,9 & 0 & 23 & 0,972 & 28,4 & 0 & 7 & 0,883 & 3,3 & 0 \\
\hline CLMI & 7 & 78 & 0,859 & $-51,7$ & 7,4 & 22 & 0,981 & 25,7 & 0 & 7 & 0,907 & 4,4 & 0 \\
\hline THLMI & 3 & 7 & 0,894 & 4,4 & 0 & - & - & - & - & - & - & - & - \\
\hline
\end{tabular}

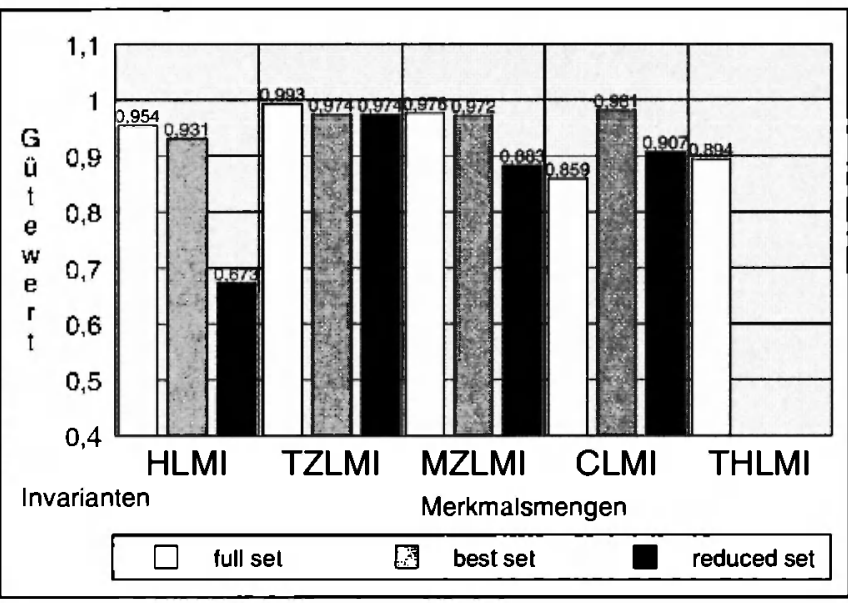

Bild 16: Klassifikationsgüten $q$ der Textur aus Texeln.

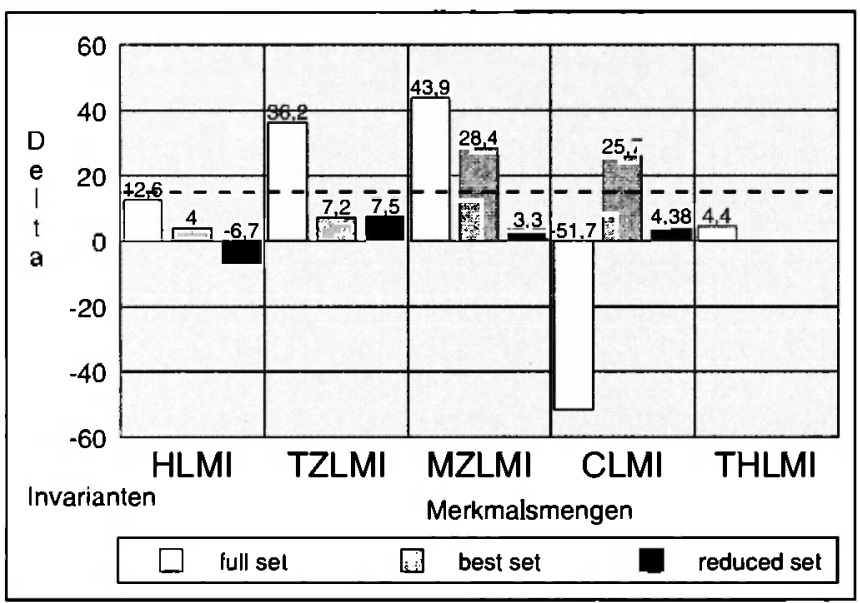

Bild 17: Rückweisungssicherheiten $\Delta$ der Textur aus Texeln.

Die exakte Auswertung der Versuche für die untersuchten Invarianten und die verschiedenen Merkmalssätze ist in Tabelle 2 dargestellt. Neben der Dimension der jeweiligen Merkmalssätze $D$, den Klassifikationsgüten $q$, den Rückweisungssicherheiten $\Delta$ ist noch der Klassifikationsfehler $e$ in $\%$ angegeben. In den Bildern 16 und 17 sind zur besseren Anschauung die Klassifikationsgüten $q$ und die Rückweisungssicherheiten $\Delta$ in Form von Balkendiagrammen dargestellt.

Die Klassifikationsgüten liegen durchweg sehr hoch. Die beiden niedrigsten Güten (reduced set HLMI, full set CLMI) gehen einher mit einer negativen Rück- weisungssicherheit. In diesen beiden Fällen war also keine fehlerfreie Klassifikation des Testdatensatzes möglich. Im Fall des full set der CLMI ist eine Klassifikation mit 78-dimensionalen Merkmalen nicht mehr sinnvoll durchführbar. Die Strichlinie in Bild 17 kennzeichnet die nach den gesammelten Erfahrungswerten notwendige Rückweisungssicherheit, um eine auch auf größeren Datensätzen sichere Fehlererkennung zu gewährleisten. Demnach können die MZLMI für die vorliegende Anwendung als die besten Merkmale bezeichnet werden.

\subsection{Schnelle Erkennung ebener Objekte}

Als zweite Anwendung soll ein Problem der ebenen Objekterkennung betrachtet werden. Es wurde das Erkennen von Puzzleteilen in beliebigen rotatorischen Lagen ausgewählt, da hier die dem Linienmomentenansatz inhärente Möglichkeit der gemeinsamen Modellierung von Objektrand und Oberflächenstruktur demonstriert werden kann. Die Bilder 5, 6 und 11 zeigen als Beispiel eine Grauwertszene, das Konturbild und die segmentierten Puzzleteile. Insgesamt sind 15 Puzzleteile in beliebigen Lagen zu unterscheiden, fehlerhafte Objekte sind in dieser Anwendung nicht zu detektieren. Die Trainingsdaten zur Berechnung der klassenspezifischen Normalverteilungen wurden aus 30 Bildern in 6 verschiedenen Winkellagen mit jeweils allen Puzzleteilen gewonnen (450 Trainingsvektoren). Getestet wurde an 6 Bildern mit Puzzleteilen in 6 verschiedenen Winkellagen (90 Testvektoren).

In Bild 18 ist die Merkmalsverteilung des gesamten Trainingsdatensatzes für das best set der MZLMI (vergleiche Tabelle 3) visualisiert. Auch hier wurde zur Projektion des 21-dimensionalen Merkmalsraumes auf 3 Dimensionen der Sammons Algorithmus ([13]) eingesetzt. Trotz der erheblichen Dimensionsreduktion bei der Projektion ist eine deutliche Clusterbildung der 15 Puzzleteileklassen zu erkennen, was die Signifikanz der Linienmomenten-Invarianten unterstreicht.

Die exakte Auswertung des Puzzlebeispiels für die untersuchten Invarianten und die verschiedenen Merkmalssätze ist in Tabelle 3 aufgeführt. Die Merkmalsdimensionen $D$, die Klassifikationsgüten $q$ und der Klassifikationsfehler $e$ in \% sind angegeben. In Bild 19 sind zur Veranschaulichung die Klassifikationsgüten $q$ als Balkendiagramm dargestellt. Auch hier liegen die Werte für $q$ durchweg sehr hoch. Eine Entscheidung für das best set als Merkmalssatz stellt für alle Invarianten 


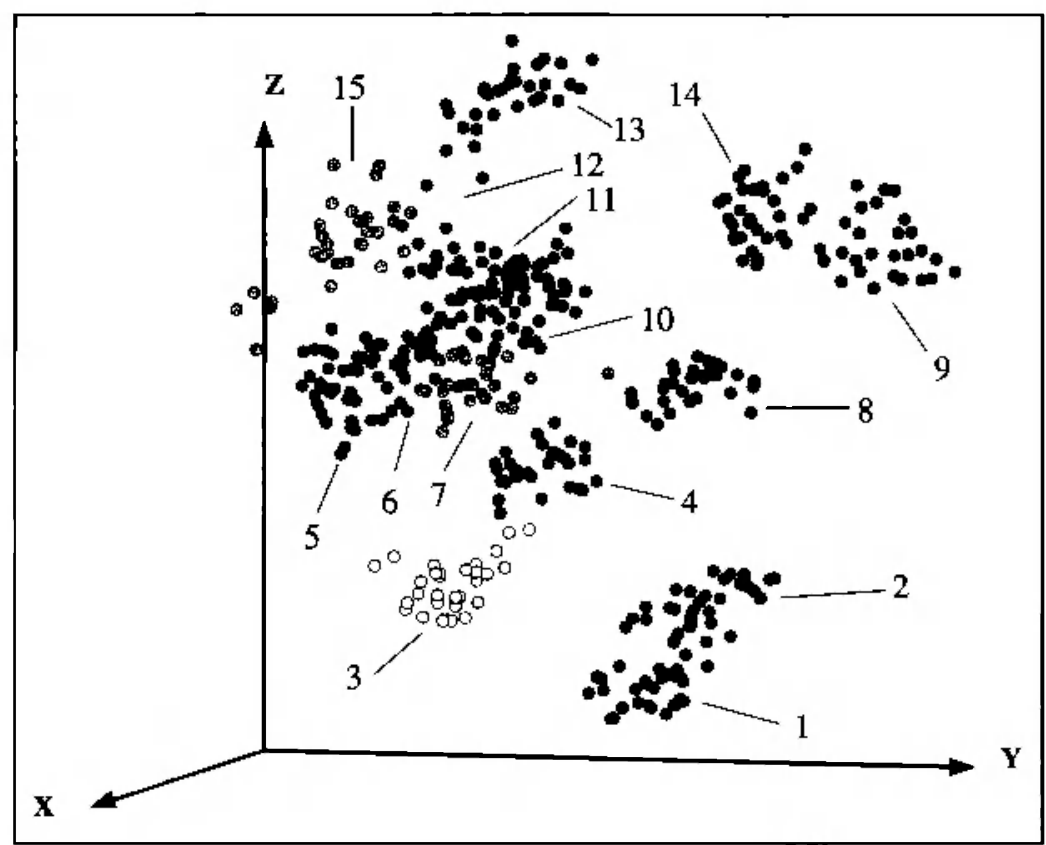

Bild 18: Visualisierung der Merkmalsverteilung des Trainingsdatensatzes (Puzzlebeispiel), 15 Klassen, Projektion von 21 auf 3 Dimensionen (Sammons Algorithmus).

Tabelle 3: Merkmalsdimension $D$, Klassifikationsgüte $q$ und Klassifikationsfehler $e$ für die untersuchten Invarianten und Merkmalssätze des Puzzle Testdatensatzes.

\begin{tabular}{|l|l|l|l|l|l|l|l|l|l|l|}
\hline \multirow{2}{*}{ Invarianten } & & \multicolumn{4}{l}{ full set } & \multicolumn{4}{l|}{ best set } & \multicolumn{3}{l|}{ reduced set } \\
\cline { 2 - 13 } & $N$ & $D$ & $q$ & $e[\%]$ & $D$ & $q$ & $e[\%]$ & $D$ & $q$ & $e[\%]$ \\
\hline HLMI & 7 & 32 & 0,964 & 0 & 17 & 0,938 & 0 & 7 & 0,898 & 0 \\
\hline TZLMI & 7 & 32 & 0,943 & 0 & 13 & 0,927 & 0 & 7 & 0,901 & 0 \\
\hline MZLMI & 7 & 32 & 0,968 & 0 & 21 & 0,967 & 0 & 7 & 0,895 & 0 \\
\hline CLMI & 7 & 78 & 0,903 & 0 & 38 & 0,971 & 0 & 7 & 0,906 & 0 \\
\hline THLMI & 3 & 7 & 0,779 & 4,4 & - & - & - & - & - & - \\
\hline
\end{tabular}

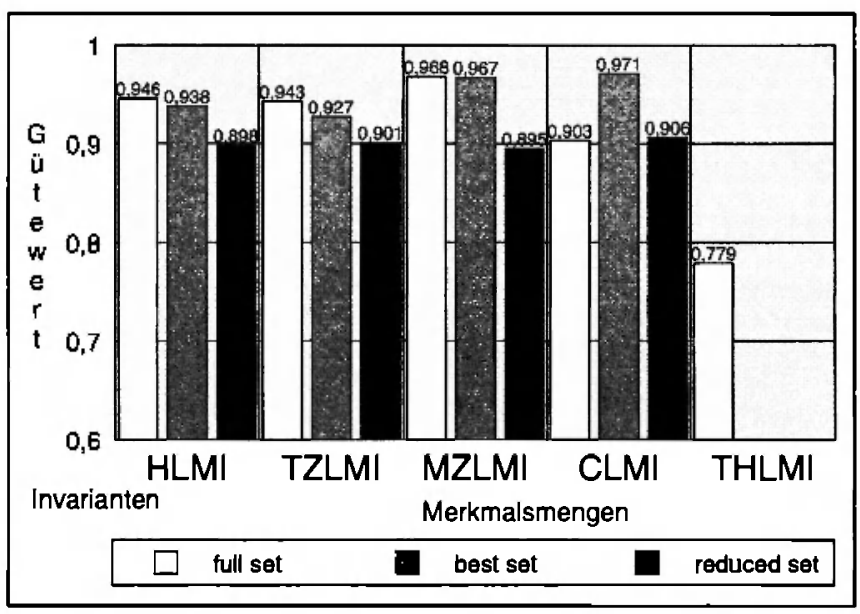

Bild 19: Klassifikationsgüten $q$ für das Erkennen der Puzzleteile.

eine gute Lösung dar. Die auf Linienmomenten bis zur Ordnung $N=3$ basierenden THLMI können den Testdatensatz nicht fehlerfrei klassifizieren (vergleiche Tabelle 3). Das für alle anderen Invarianten auf 7 Merkmale reduzierte reduced set kann zwar fehlerfrei klassifizieren, liegt jedoch in der Klassifikationsgüte deutlich unter den anderen Merkmalssätzen.

\section{Zusammenfassung und Ausblick}

Der Beitrag stellt eine neue Methode zur konturbasierten Bildverarbeitung in Echtzeit vor. Ausgehend von den aus der Literatur bekannten Flächenmomenten wird die Überführung dieser Ansätze auf Konturen in Form von Linienmomenten durchgeführt. Die dem Linienmomentenansatz inhärente Fähigkeit, beliebige Sammlungen nichtzusammenhängender Konturfragmente ohne geschlossenen Rand als Gesamtheit modellieren zu können, erlaubt eine entsprechend flexible Definition von Konturobjekten. Der Linienmomentenansatz ist somit flexibler einsetzbar und erfordert weniger Vorverarbeitungsaufwand als andere konturbasierte Methoden, wie z.B. Fourierdeskriptoren.

Basierend auf der Polygonapproximation von Konturen wird auf eine effiziente Berechnung der geometrischen Linienmomente eingegangen. Die Anwendung des Linienmomentenkonzepts auf andere Momententypen eröffnet dem Ansatz eine große Menge bestehender Theorien zur Berechnung von bezüglich der Objektverdrehung invarianten Merkmalen. In Anwendungen zur Inspektion von Texturen aus Texeln und der schnellen Erkennung ebener Objekte wird die Signifikanz und Güte der Merkmale herausgestellt.

Durch die Fülle existierender Theorien im Bereich der Flächenmomente sind weiterführende Untersuchungen zur Übertragung der Ansätze auf das Linienmomentenkonzept sinnvoll (z.B. im Bereich der 3D Invariantenbildung und Objekterkennung). Eine Herleitung des in den verschiedenen Momententypen modellierten Informationsgehalts über Objektformen hat für Flächenmomente nur vereinzelt stattgefunden. Entsprechende Betrachtungen wurden für Linienmomente noch nicht durchgeführt.

Mit der Methode der Linienmomente im Zusammenhang mit den aufgezeigten Verfahrensschritten steht dem Anwender ein leistungsstarkes und besonders schnelles Konzept zur Analyse strukturierter Bildszenen zur Verfügung.

Die Arbeit wurde gefördert im Rahmen des BMBF Verbundprojekts TEXAUGE von der ISRA Systemtechnik GmbH, Darmstadt.

\section{Literatur}

[1] Lambert, G., Gao, H., Hohm, K. und Amelung, J.: Linienmomente und Invarianten zur Echtzeitverarbeitung vektorisierter Konturen. In Mustererkennung 1995, 17. DAGM Sympơsỉum, Sèitèn 570-577. Springer, Septembèr 1995.

[2] Lambert, $G$. und Noll. J.: Discrimination properties of invariants using the line moments of vectorized contours. In 
13th International Conference on Pattern Recognition, Volume II, Pattern Recognition and Signal Analysis, Seiten 735-739. IEEE Computer Society Press, August 1996.

[3] $H u, M .-K$.: Visual Pattem Recognition by Moment Invariants. IRE Trans. on Information Theorie, 8:179-187, 1962.

[4] Teh, C.-H. und Chin, R. T.: On Image Analysis by the Methods of Moments. IEEE Trans. on Pattern Analysis and Machine Intelligence, 10(4):496-513, 1988.

[5] Belkasim, S. O., Shridhar, M. und Ahmadi, M.: Pattern Recognition With Moment Invariants: A Comparative Study And New Results. Pattern Recognition, 24(12):1117-1138, 1991.

[6] Bailey, $R$. R. und Srinath, M.: Orthogonal Moment Features for Use with Parametric and Non-Parametric Classifiers. IEEE Trans. on Pattern Analysis and Machine Intelligence, 18(4):389-399, 1996.

[7] Liao, S. X. und Pawlak, M.: On Image Analysis by Moments. IEEE Transactions on Pattern Analysis and Machine Intelligence, 18(3):254-266, 1996.
[8] Teague, $M . R$.: Image analysis via the general theory of moments. J. Opt. Soc. Am., 70(8):920-930, 1980.

[9] Abu-Mostafa, Y. S. und Psaltis, D.: Recognitive Aspects of Moment Invariants. IEEE Trans. on Pattern Analysis and Machine Intelligence, 6(6):698-706, 1984.

[10] Abu-Mostafa, Y. S. und Psaltis, D.: Image Normalisation by Complex Moments. IEEE Trans. on Pattern Analysis and Machine Intelligence, 7(1):46-55, 1985.

[11] Fukunaga, K.: Introduction to Statistical Pattern Recognition Academic Press, Boston, 1990.

[12] Lambert, G., Amelung, J. und Ersü, E.: Konzepte zur Auslegung von Echtzeit-Bildverarbeitungssystemen für die Qualitätssicherung am Beispiel der Inspektion von Texturen. at Automatisierungstechnik, Oldenbourg, 45(5):209-217, 1997.

[13] Sammon, J. W.: A Nonlinear Mapping for Data Structure Analysis. IEEE Trans. on Computers, C-18(5):401-409, 1969.

Manuskripteingang: 5. Mai 1997.

Verfügbar unter

lediglich die vom Gesetz vorgesehenen Nutzungsrechte gemäß UrhG 